\title{
Economic Assessment of 4 Approaches to the Diagnosis and Initial Treatment of Sleep Apnea
}

\author{
Dawn M Bravata MD, Nancy Lightner PhD, H Klar Yaggi MD MPH, and Edward J Miech EdD
}

\begin{abstract}
BACKGROUND: A dilemma faced by health-care administrators is that need greatly outstrips capacity for diagnosing and treating sleep apnea, with such decisions carrying significant economic consequences. Our objective was to develop an economic model to estimate the relative costs of 4 approaches for diagnosis and initial treatment of sleep apnea. METHODS: The analysis consisted of developing a mathematical model depicting possible diagnostic and treatment approaches to the care of patients with sleep apnea; developing 4 clinical scenarios to describe distinct approaches to the management of sleep apnea patients (in-laboratory, unattended, direct-to-autotitrating PAP [auto-PAP], and mixed); and identifying costs associated with each scenario. We created a hypothetical cohort of 1,000 patients with $85 \%$ prevalence of sleep apnea to generate cost estimates. RESULTS: The driver of per-patient costs was the total number of sleep studies, which varied widely across scenarios: from 425 for the direct-to-auto-PAP approach to 1,441 in the unattended approach. The scenarios also differed in per-patient costs: Per-patient costs excluding facility startup costs were $\mathbf{\$ 4 5 6}$ for direct-to-auto-PAP, $\$ 913$ for in-laboratory, $\$ 991$ for mixed, and $\$ 1,090$ for unattended. CONCLUSIONS: Approaches to diagnosing and treating sleep apnea that emphasized early application of auto-PAP had lower per-patient costs. Key words: sleep apnea; economic model; health-care capacity; positive airway pressure; unattended polysomnography; health services research. [Respir Care 2018;63(1):50-61. (c) 2018 Daedalus Enterprises]
\end{abstract}

\section{Introduction}

Health-care administrators and policymakers face a basic dilemma when identifying patients with sleep apnea: Need greatly outstrips capacity. Sleep apnea is a commonly recognized medical problem in the United States, yet an estimated $70-80 \%$ of patients with the condition remain undiagnosed. ${ }^{1-4}$ Because sleep apnea is associated

\footnotetext{
Drs Bravata, Lightner, and Miech are affiliated with the Veterans Affairs Center for Applied Systems Engineering (VA-CASE), Indianapolis, Indiana. Drs Bravata and Miech are also affiliated with the Department of Veterans Affairs Health Services Research and Development (HSR\&D) Center for Health Information and Communication (CHIC), Richard L Roudebush Veterans Affairs Medical Center, Indianapolis, Indiana; the Department of Internal Medicine, Indiana University School of Medicine, Indianapolis, Indiana; and the Regenstrief Institute, Indianapolis, Indiana. Dr Miech is also affiliated with the Department of Emergency Medicine, Indiana University School of Medicine, Indianapolis, Indiana. Dr Yaggi is affiliated with the Medicine Service, Veterans Affairs Connecticut Healthcare System, West Haven, Connecticut and the Department of Internal Medicine, Yale University School of Medicine, New Haven, Connecticut.
}

with a variety of poor patient outcomes, ${ }^{5,6}$ awareness of the importance of diagnosing sleep apnea has become much more common in recent years, with referrals for polysomnography, the accepted standard method for diagnosing sleep apnea, increasing 12-fold worldwide over the past decade. ${ }^{1}$ Sleep laboratory capacity has not accompanied the dramatic increase in demand, contributing to widespread underdiagnosis and undertreatment of sleep apnea. ${ }^{7}$

\footnotetext{
This project was supported by Department of Veterans Affairs Health Services Research and Development Service (VA HSR\&D) Grant IIR06-233-2. The authors have disclosed no conflicts of interest.

Supplementary material related to this paper is available at http:// www.rcjournal.com.

Correspondence: Dawn M Bravata MD, Richard L Roudebush VA Medical Center, HSR\&D Mail Code 11H, 1481 West 10th Street, Indianapolis, IN 46202. E-mail: dawn.bravata2@va.gov.
}

DOI: $10.4187 /$ respcare. 05355 
The Veterans Health Administration (VA), the single largest health-care provider in the United States, provides a large-scale example of the tremendous challenges associated with the diagnosis of obstructive sleep apnea (OSA). The VA estimates that 1.5 million veterans have OSA, yet in 2001, there were only 148 beds in VA sleep laboratories across the United States serving a total of about 6,000 patients. ${ }^{8,9}$ Of the total number of patients with OSA in the general veteran population, an estimated $95 \%$ of veterans with OSA remain undiagnosed..$^{10}$

This disparity between need and capacity is particularly striking within the VA because the prevalence of OSA among veterans is approximately 4-fold higher than in the general United States population. ${ }^{10}$ This is because factors associated with OSA, such as older age, obesity, male sex, and alcohol use are all relatively common within the VA patient population. ${ }^{10}$ Given the bottleneck of inadequate capacity in existing sleep laboratories, additional approaches to diagnosing and treating OSA may need to be implemented, specifically approaches that are accurate, cost-efficient, and scalable. We sought to construct an economic model to estimate the costs of building sleep medicine programs for the diagnosis and initial treatment of OSA in the VA to address this fundamental constraint in current capacity using 4 different clinical approaches. Our focus was on comparing the relative costs of each scenario and the drivers of those costs. We maintained the perspective of the health-care system in the construction of the economic model.

\section{Methods}

The methods consisted of 3 components (1) developing a mathematical model depicting possible diagnostic and treatment approaches to the care of patients with OSA, (2) developing clinical scenarios to describe distinct approaches to the management of patients with OSA, and (3) identifying the costs associated with each of the scenarios.

\section{Model Construction}

Model development involved constructing a flow model describing components of a sleep medicine program and identifying costs for each elements of the flow model.

Flow Model Development. The goal was to develop a flow model that was comprehensive in terms of the diagnosis and treatment of OSA, including all possible elements of a sleep medicine program as it would be applied to a patient with possible OSA. The clinical care of patients with non-OSA disorders (eg, narcolepsy) was outside of the scope of the project.

\section{QUICK LOOK}

\section{Current knowledge}

While OSA is a commonly recognized medical problem in the United States, a large majority of patients with the condition remain undiagnosed. Because OSA is associated with a variety of poor patient outcomes, awareness of the importance of diagnosing OSA has become much more common, with referrals for polysomnography sharply increasing over the past decade. Sleep laboratory capacity has not accompanied the dramatic increase in demand, contributing to widespread under-diagnosis and under-treatment of OSA.

\section{What this paper contributes to our knowledge}

In a newly-developed mathematical model designed to generate evidence-based cost estimates of 4 possible diagnostic and treatment approaches to the care of patients with OSA (in-laboratory, unattended, direct-toauto-PAP, and mixed), the main driver of per-patient costs was the total number of sleep studies, with this total varying widely across scenarios. Approaches that emphasized early application of auto-PAP had lower per-patient costs.

The flow model included elements and personnel related to the diagnosis of OSA and to the subsequent treatment of patients with diagnosed OSA. The most commonly used and widely accepted procedure used to diagnose OSA is polysomnography (PSG). PSG may be performed in a sleep laboratory or in a non-laboratory setting and may be attended or unattended. Laboratory-based PSG is considered the accepted standard for OSA diagnosis. ${ }^{10,11}$ In-laboratory PSG involves having a patient spend the night in a sleep laboratory, where a technician monitors the patient using sensors that monitora broad range of sleep, cardiovascular, and respiratory-related elements. If technicians detect OSA during the polysomnography, they may apply a positive airway pressure (PAP) device to identify the pressure at which the OSA is best treated; this procedure is referred to as a split-night study. The results of a PSG are reviewed by a scorer, and then a sleep medicine physician reviews the scored results and makes treatment and follow-up recommendations. The laboratory-based PSG yields results that are highly accurate but requires a sleep laboratory and significant personnel resources. Some patients complain that they cannot sleep normally in the unfamiliar and highly monitored environment of the sleep laboratory, which may lead to a reduction in observed hours of sleep during the in-laboratory PSG. ${ }^{12}$ Alternatives to in-laboratory PSG include portable PSG, which is usu- 
ally conducted in a patient's home without overnight monitoring by a technician and thus referred to as unattended.

Although the treatment of OSA includes behavioral recommendations (eg, weight loss), the cornerstone of therapy involves the use of PAP while sleeping. PAP may be applied using a fixed pressure setting or using autotitrating settings. Fixed pressure PAP requires in-laboratory PAP titration by a technician. Auto-titrating PAP (auto-PAP) machines eliminate respiratory disturbances by varying the pressure delivered, depending on the patients' respiratory efforts. Auto-PAP compared with fixed pressure PAP is equally efficacious but is not appropriate for all patients with OSA (eg, contraindicated for patients with congestive heart failure). ${ }^{13-15}$ Auto-titrating PAP for treatment makes it possible to shift the principal location for managing OSA from the sleep laboratory to the patient's home.

Members of the research team (NL and DMB) developed the initial flow model, which was then iteratively revised in consultation with the VA Sleep Field Advisory Committee. The committee determined the default staff members for each patient contact (ie, whether a particular patient contact should involve a nurse or a physician or a respiratory therapist) and the average duration of each contact. Figure 1 displays the final flow model. Each box represents an episode of patient contact (eg, PAP initiation). The diamond shapes indicate a decision point within patient flow (eg, high residual apnea-hypopnea index [AHI] with PAP). Two paths emanate from each diamond, a "yes" and a "no" branch, based on the answer to the decision question. This flow model resulted in a total of 23 combinations of diagnosis paths, including direct-to-autoPAP, unattended study, polysomnography, and in-lab titration possibilities.

Cost Allocations. The VA Office of Construction and Facilities Management provided per square foot costs of renovation and operating costs for facility space. The Office of Construction and Facilities Management periodically revises these cost data, which are used for planning but not budgeting purposes. The economic model built for this study included the cost of renovation, which was classified into three categories: light if renovation involved the removal and replacement of up to approximately $30 \%$ of the finishes and systems; medium if removal and replacement of $31-67 \%$ of the existing space was indicated, and total renovation if $>67 \%$ removal and replacement was required.

Sleep laboratories consist of rooms where patients sleep and a monitoring station where the night sleep technician observes the patients. One monitoring station (150 square feet) can oversee up to 4 sleep study rooms of 210 square feet each. To identify only the cost of patient care, the model included the light renovation cost needed for the recommended number of sleep study rooms and associated monitoring sta- tions at a rate of 4 study rooms to 1 monitoring station. We included the cost of renovation as a startup cost, amortized over $5 \mathrm{y}$. This analysis did not include the cost of examination rooms, because our baseline-case assumption was that a facility already had adequate examination rooms. An annual operating expense was included for the needed sleep study and monitoring rooms at the local facility rate specified by the Office of Construction and Facilities Management.

Costs included in the per-patient costs included: startup costs annualized over $5 \mathrm{y}$ for the recommended sleep study rooms; facility operating costs, depending on the amount of facility space renovated; sleep medicine personnel involved in patient care; and PSG supplies. We derived the average cost of PSG supplies based on actual calendaryear 2007 acquisition costs $^{16}$ as follows: $\$ 234$ for an unattended PSG, \$200 for a full-night in-laboratory PSG, and $\$ 305$ for either an in-laboratory split-night or titration study.

Table 1 contains personnel costs for one full-time employee equivalent used in all scenario calculations. The salaries included in the model were obtained from fiscal year 2012 base pay rates, specifically the mid-point of the starting salary range for staff at the Indianapolis VA facility. The Indiana facility was chosen because locality pay for the Midwest is intermediate between the observed extreme values (eg, highest in Northeast and California and lowest in the South). The base pay amounts do not include benefit pay or locality pay. Locality pay is used in the VA to compensate staff in varying geographic regions. For example, staff in the Northeast generally receive considerably more locality pay than staff in the same position in the South.

Table 2 displays the personnel and duration assigned to each patient contact. The full-time employee equivalent utilization rate was $85 \%$ to account for vacation, sickness, education, and other reasons for time off. The staffing costs were based on hiring only for the recommended effort. For example, if a given scenario required $65 \%$ of a physician, only $65 \%$ of the physician total costs $(\$ 223,626 \times 0.65)$ were included in the analysis. In reality, facilities are more likely to hire a 1.0 full-time employee equivalent physician in this situation and assign that individual additional clinical duties. Rounding up in this analysis was avoided because of its potential to obscure differences between scenarios. Staffing costs were based on the need for 0.25 full-time employee equivalent for a physician to direct the sleep program and 1.00 fulltime employee equivalent for a sleep medicine nurse unit manager. Polysomnographic scoring was included in the analysis as requiring an initial review by a scorer $(2 \mathrm{~h}$ per in-laboratory PSG and $1 \mathrm{~h}$ per unattended study) and confirmation by a physician ( $1 \mathrm{~h}$ per in-laboratory PSG and 30 min per unattended study). PAP titration studies were 


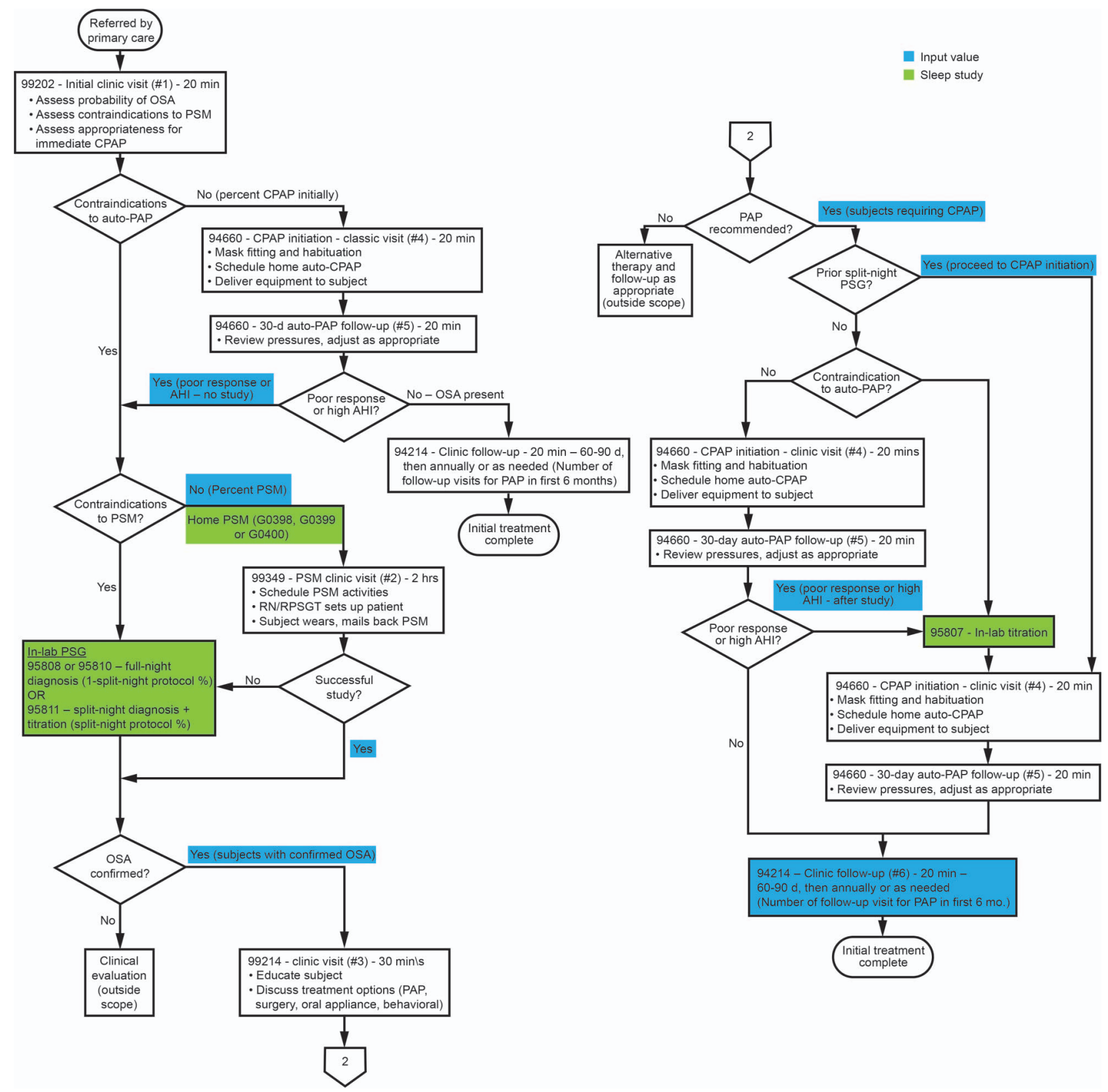

Fig. 1. Sleep Medicine Program flow chart. PSM = portable sleep monitoring, $\mathrm{AHI}=$ apnea-hypopnea index, OSA = obstructive sleep apnea, PSG = polysomnography.

included in the analysis as requiring $1 \mathrm{~h}$ of effort by the scorer and 30 min of physician time.

\section{Clinical Scenarios}

We considered 4 scenarios for OSA diagnosis and treatment to illustrate a wide variety of approaches to designing a sleep medicine program. Our goal was to create distinct approaches to the diagnosis and treatment of OSA and then identify the key drivers of costs across these 4 scenarios. We sought to design relatively extreme scenarios that may not represent current standard practice but that illustrate how differences in sleep medicine program design might influence costs.

All of the scenarios used the same set of baseline-case assumptions, which drew upon recommendations of the VA Field Advisory Committee as follows: $85 \%$ of patients who were referred to a sleep medicine program would have OSA (ie, the prevalence of OSA in the population was $85 \%$ ); $80 \%$ of unattended studies would have ade- 
Table 1 Personnel Costs

\begin{tabular}{lrr}
\hline \hline \multicolumn{1}{c}{ Personnel } & $\begin{array}{c}\text { Base } \\
\text { Salary }\end{array}$ & $\begin{array}{c}\text { Total } \\
\text { Cost* }\end{array}$ \\
\hline $\begin{array}{l}\text { Required } \\
\quad \text { Sleep physician (medical director, 0.25 FTEE) }\end{array}$ & $\$ 150,000$ & $\$ 55,907$ \\
$\quad$ Nurse unit manager (1.00 FTEE) & $\$ 65,000$ & $\$ 96,905$ \\
Variable time-allocated, depending on approach & & \\
$\quad$ Sleep medicine physician & $\$ 150,000$ & $\$ 223,626$ \\
$\quad$ Sleep medicine nurse & $\$ 65,000$ & $\$ 96,905$ \\
Night sleep technician & $\$ 40,000$ & $\$ 59,634$ \\
Day respiratory therapist & $\$ 55,000$ & $\$ 81,996$ \\
Polysomnography scorer & $\$ 50,000$ & $\$ 74,542$ \\
Office assistant & $\$ 35,000$ & $\$ 52,179$ \\
& & \\
* Total costs include locality pay and 30\% fringe-benefit cost. & & \\
FTEE = full-time employee equivalent & & \\
\hline
\end{tabular}

quate data for interpretation, with the remaining $20 \%$ requiring an additional follow-up in-laboratory study; $100 \%$ of patients with OSA would be referred for PAP; $100 \%$ of patients with OSA receive a 20-min visit with a physician to review the diagnosis and treatment options; $100 \%$ of patients receiving CPAP have a 20-min visit with a day sleep technician for mask fitting and habituation and then a 20-min visit with a day sleep technician at $30 \mathrm{~d}$ postinitiation for troubleshooting and follow-up; $20 \%$ of patients would have a contraindication to auto-PAP or a poor response to auto-PAP (eg, high residual AHI on auto-PAP), and these patients would require in-laboratory titration; PAP devices that are provided to patients who do not tolerate or use them are not refurbished for use by other patients; and $40 \%$ of in-laboratory PSG patients who were positive for OSA would have a split-night study that included PAP titration. These rates are included in the flow model depicting each scenario in Figures 2-5.

Scenario 1: In-Laboratory. The in-laboratory approach (see Fig. 2) involves patients with suspected OSA receiving an in-laboratory PSG. Patients diagnosed with OSA receive either a split-night study or a separate in-laboratory PAP titration. This approach does not use auto-PAP or unattended PSG.

Scenario 2: Unattended. In the unattended approach, the patient conducts the sleep study without monitoring by a technician. Health-care organizations use 2 main strategies for unattended polysomnography: sending staff to patients' homes to set them up with the equipment or requiring patients come to a facility for equipment and instructions. Because the latter is the more common approach used in the VA, we modeled that scenario, employing type 3 portable sleep monitoring equipment. In the unattended approach (see Fig. 3), patients with suspected OSA go to a health-care facility, where they are set up for a subsequent unattended, in-home PSG. Patients return home, sleep using the equipment, and then mail it back to the facility. Patients diagnosed with OSA are given autoPAP; some of these patients will have a poor response to auto-PAP and will thus require in-laboratory CPAP titration. Patients who are negative for OSA are referred for

Table 2 Personnel Assignment for Each Activity

\begin{tabular}{lll}
\hline \hline \multicolumn{1}{c}{ Activity Description } & \multicolumn{1}{c}{ Assigned Personnel } & Duration \\
\hline Assessment of OSA probability & Physician & $20 \mathrm{~min}$ \\
Unattended PSG visit & Day respiratory therapist & $2 \mathrm{~h}$ \\
OSA clinic visit to discuss treatment options & Physician & $20 \mathrm{~min}$ \\
PAP initiation & Day respiratory therapist & $20 \mathrm{~min}$ \\
30-d auto-PAP follow-up & Day respiratory therapist & 20 min \\
Additional PAP follow-up visits (2) & Registered nurse \\
Scoring of PSG & Lab scorer \\
& Physician \\
Scoring of unattended PSG & Lab scorer \\
& Physician \\
Scoring of titration studies & Lab scorer \\
& Physician \\
In-laboratory PSG & Night sleep technician \\
In-laboratory PAP full-night titration & Night sleep technician \\
In-laboratory split-night PAP titration & Night sleep technician \\
& \\
\hline The assignments refer to the type of person conducting an activity and the amount of time spent in each activity. The model could be modified as to whether or not any given activity was included \\
in the sleep medicine program design.
\end{tabular}




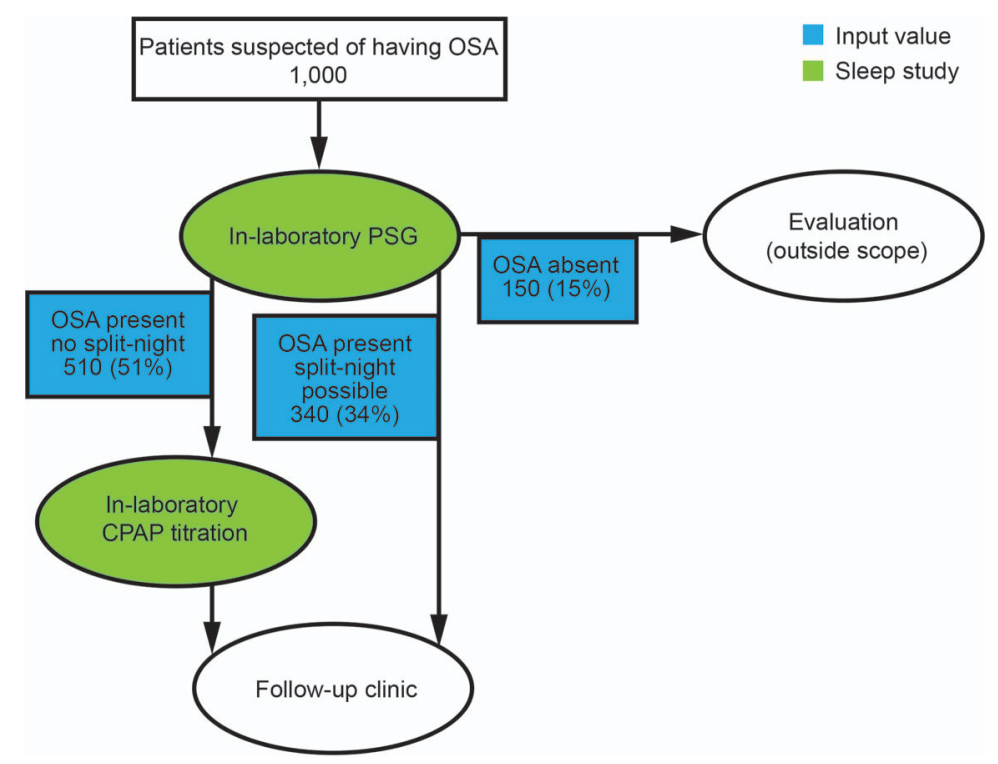

Fig. 2. In-laboratory approach.

in-laboratory PSG if clinical suspicion for OSA is high; otherwise, no further PSG is needed. If patients do not tolerate auto-PAP, they will receive in-laboratory CPAP titration.

Scenario 3: Direct-to-Auto-PAP Approach. In the direct-to-auto-PAP approach (see Fig. 4), patients with suspected OSA and no obvious contraindications to auto-PAP are given an auto-PAP machine and instruction on its use. The physician may use screening tools, such as the Berlin Questionnaire or the Sleep Apnea Clinical Score, ${ }^{17,18}$ during the initial examination to identify suspected OSA. The auto-PAP data are reviewed after a fixed period of time (eg, $7 \mathrm{~d}$ ) and considered positive based on elevations of the residual AHI (ie, AHI $\geq 5$ events/h) or the median pressure (ie, $\geq 6 \mathrm{~cm} \mathrm{H}_{2} \mathrm{O}$ ); positive patients retain their machines and receive clinic follow-up. ${ }^{19,20}$ If the auto-PAP is negative, then the patient is referred to in-laboratory PSG with split-night titration if possible. If a patient has a poor response to auto-PAP (eg, high residual AHI values), he or she is referred for in-laboratory CPAP titration. Patients suspected of having contraindications to auto-PAP are referred for an in-laboratory PSG. Because VA patients are not subject to reimbursement requirements of the Centers for Medicare and Medicaid Service or private insurance companies, this approach is potentially feasible (albeit not commonly used) in VA.

Scenario 4: Mixed Approach. In the mixed approach (see Fig. 5), half of the patients with suspected OSA are set up for unattended PSG and follow the course described in the unattended approach, and the other half are referred for in-laboratory testing and follow the in-laboratory ap- proach. In practice, patients with a high probability of OSA who live far away from a sleep laboratory or decline to have an in-laboratory study would be candidates for unattended PSG.

\section{Model Interrogation to Identify Costs per Scenario}

To demonstrate the economic effect of each of the scenarios, we created a hypothetical cohort of veterans $(N=1,000)$ who were referred to a VA sleep medicine program for the purpose of screening and management of OSA. We compared the estimated costs and timeline for caring for the 1,000-patient cohort across the different scenarios. The scenarios differed in 2 values: the proportion of patients who received PAP initially and the proportion of patients who receive unattended studies. For example, in the direct-to-auto-PAP approach, $100 \%$ of patients initially get assigned to PAP. The same flow model applied across all scenarios, with changes in the proportion of patients in the hypothetical cohort of 1,000 patients who receive certain program elements. The baseline assumption input values described earlier were entered into the model to identify the costs for each of the scenarios. The model identified the facility space and personnel time needed for the 4 scenarios, and these requirements were used to calculate costs. By comparing the model outputs for each of the 4 scenarios, we identified key drivers of costs.

\section{Results}

The scenarios varied considerably in terms of the total number of sleep studies that were ultimately required by each approach, ranging from 425 for direct-to-auto-PAP to 


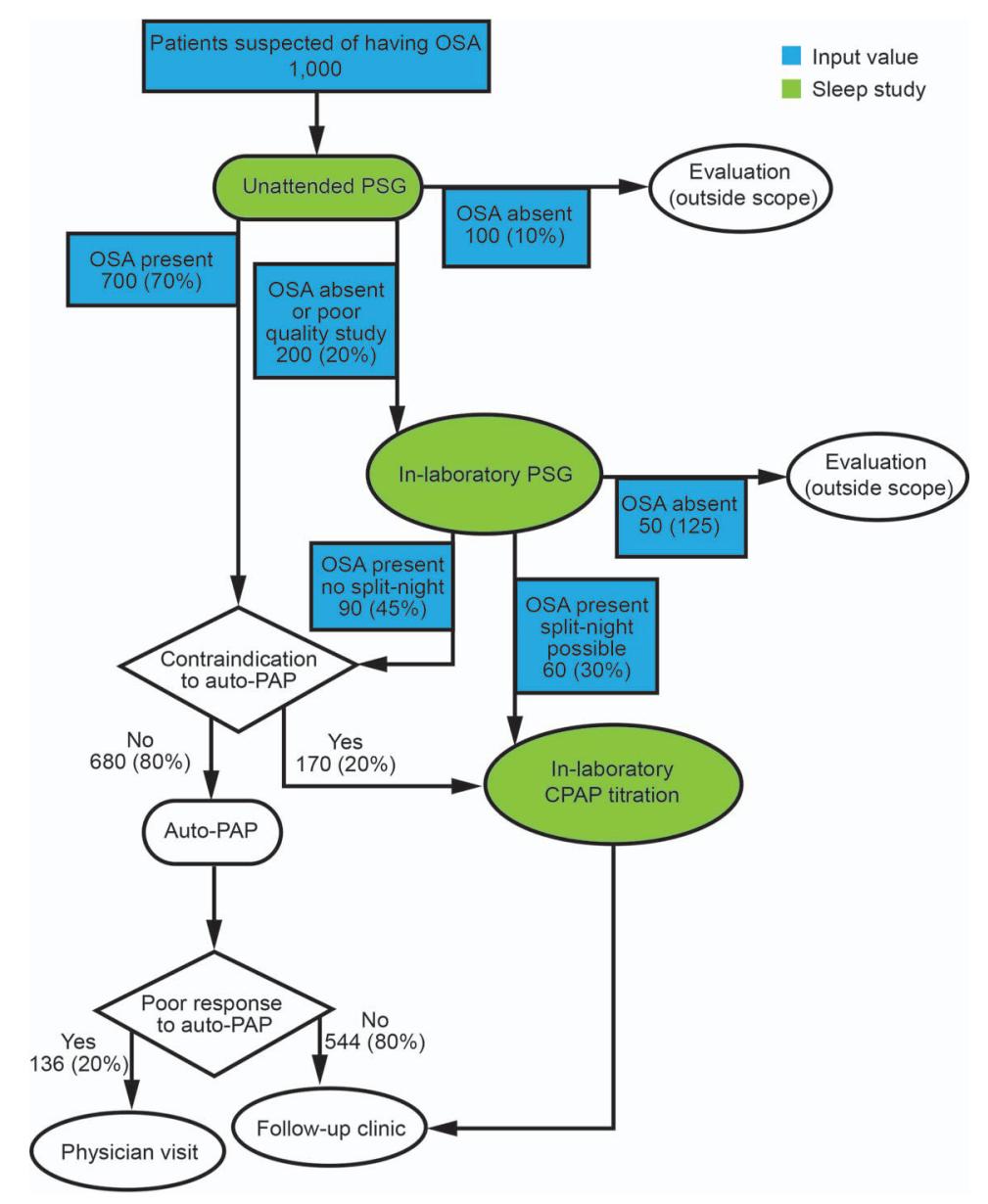

Fig. 3. Unattended approach.

1,441 for unattended (see Table 3). The number of sleep studies drove staffing costs because of the need to score and review studies, which applies to both unattended and in-laboratory PSGs. The lowest personnel costs were observed for the direct-to-auto-PAP approach. Personnel costs for the other 3 approaches were relatively similar and approximately 2 times higher than those for the direct-toauto-PAP approach. The number of sleep studies also drove supply costs, which were lowest for direct-to-auto-PAP and highest for unattended $(\$ 99,287$ vs $\$ 351,027$ for the 1,000-patient cohort; see Table 3).

Table 3 also shows the number of additional rooms needed for each scenario. The in-laboratory approach facility cost was highest, almost 3 times the facility cost of the direct-to-auto-PAP and unattended approaches.

Per-patient costs differed by scenario: $\$ 456$ for the direct-to-auto-PAP approach, $\$ 913$ for the in-laboratory approach, $\$ 991$ for the mixed approach, and $\$ 1,090$ for the unattended approach, excluding facility startup costs. Appendix A (see the supplementary materials at http:// www.rcjournal.com) provides a flow diagram for an approach that minimized cost per patient.
Table 4 displays the cost results of varying the success rate of unattended studies (ie, the proportion of studies with data of sufficient quality to score the study) from 50 to $100 \%$. The cost per patient ranged from $\$ 973$ for the extremely optimistic case, where $100 \%$ of unattended studies produced excellent data sufficient for scoring, to $\$ 1,261$ for the extremely pessimistic case of data failure in $50 \%$ of unattended studies. There was less per-patient cost variability within the range of more typically reported values of $80-95 \%$ data success (see Table 4 ).

\section{Discussion}

The in-laboratory, unattended, and direct-to-auto-PAP approaches represent a wide variety of configurations for sleep medicine programs. Comparison across these scenarios provided insights into differential costs across approaches as well as key drivers of those costs. Model results indicated that drivers of per-patient cost were personnel costs and facility operations costs related to both the number and type of PSGs (attended vs unattended). The direct-to-auto-PAP approach had the lowest cost per 


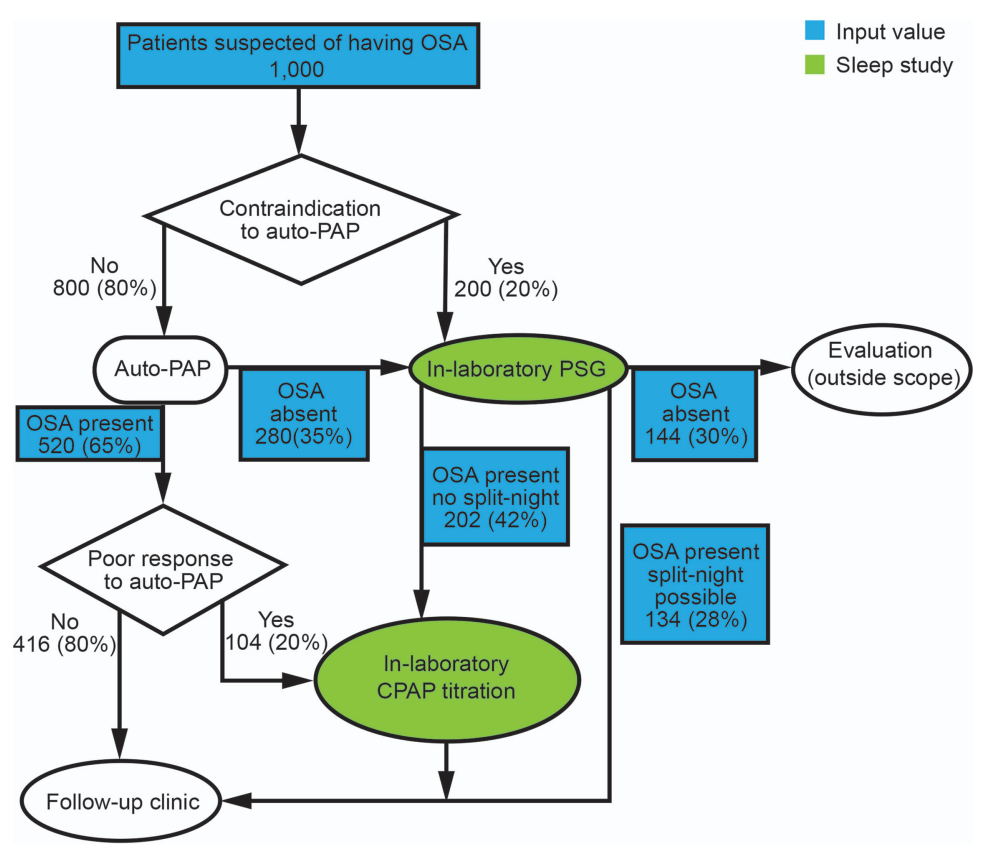

Fig. 4. Direct-to-auto-PAP approach.

patient (\$590), and the in-laboratory approach had the highest cost per patient $(\$ 1,418)$ when facility startup costs were included. The in-laboratory approach required fewer PSGs overall than the unattended or mixed scenarios, but the in-laboratory PSGs were more expensive than the unattended PSGs.

Our findings illustrate the importance of explicitly specifying and considering various facility costs in economic evaluations of health-care programs. For example, the inlaboratory approach was less expensive than either the mixed or unattended approaches when facility startup costs were excluded, but it was more expensive than both of these approaches when facility costs were included. Prior economic evaluations regarding alternative approaches to the diagnosis of patients with OSA have generally compared 2 or more approaches from a per-patient perspective and have not considered facility-level costs. ${ }^{21,22}$ They have all found that the per-patient costs (or charges) for in-laboratory PSG were higher than for in-home $\mathrm{PSG}^{21-23}$; however, the differences in costs vary from approximately $20 \% 22,23$ to $400 \%^{21}$ increase over the in-home cost. Kim et $\mathrm{al}^{23}$ included an assessment of the provider perspective in their cost analysis, which included labor costs, supplies, equipment, and facility rental costs. They found that an in-laboratory approach was $13 \%$ more expensive than an in-home approach $(\$ 1,809$ vs $\$ 1,599)$ when only patient costs (in an analysis the authors referred to as the payer perspective) were included but that the in-laboratory approach was $2 \%$ less expensive than an in-home approach when facility costs were included ( $\$ 1,697$ vs $\$ 1,736$; an analysis the authors referred to as the provider perspective). ${ }^{23}$ The find- ing by Kim et $\mathrm{al}^{23}$ similarly indicates the importance of considering facility costs in the economic assessment of alternative diagnostic approaches, but it is in the opposite direction from our finding. The Kim et al study ${ }^{23}$ was based on a randomized controlled trial and observed that $41 \%$ of the subjects who were randomized to the in-home group required in-laboratory PSG; this is twice the rate used in our model. ${ }^{23}$ The rate used in the current study was based on expert opinion and reflected the current clinical experience of VA sleep medicine physicians who have experience with the application of unattended polysomnography in patients' homes.

In addition to the focus on including facility costs and the facility perspective, the current study advances the existing literature by including a greater spectrum of alternative sleep medicine program configurations than the prior studies. Specifically, the inclusion of a direct-toauto-PAP approach and the mixed approach is, to our knowledge, a novel contribution. Our objective in designing the clinical scenarios was to provide a wide range of alternatives to identify cost differences and drivers, not to promote any particular clinical approach. The majority of sleep medicine programs in the United States do not currently employ a direct-to-auto-PAP approach. In-laboratory PSG provides diagnostic information that may be of importance to the care of an individual patient (eg, periodic limb movements) and may be indicated for patients when the clinical question extends beyond simply making or excluding the diagnosis of OSA. Given an OSA prevalence of $85 \%$ for most VA sleep medicine programs, however, the direct-to-auto-PAP approach may provide 


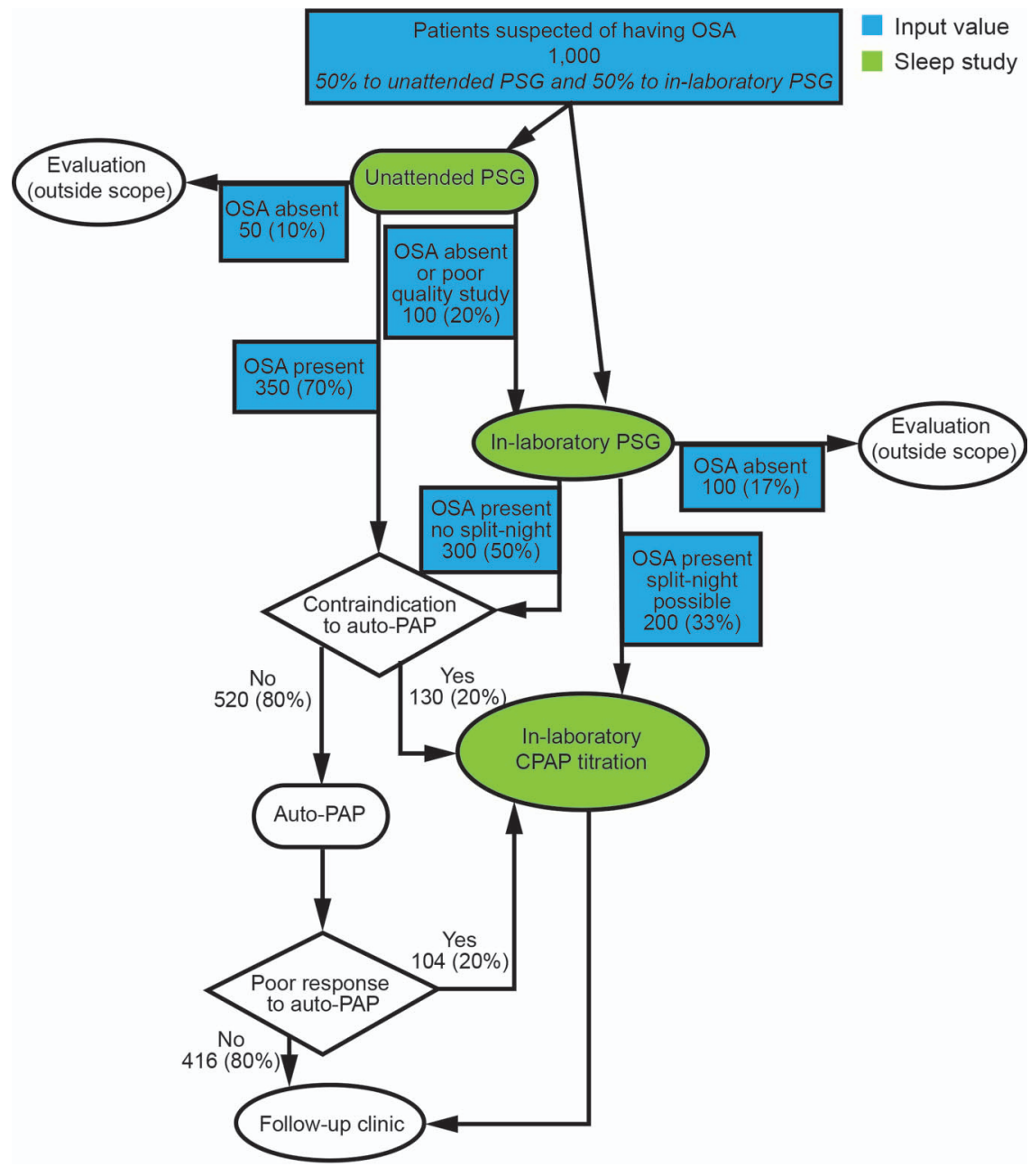

Fig. 5. Mixed approach.

treatment to patients with minimal delay and lowest overall cost for the large majority of patients who will require PAP therapy. The direct-to-auto-PAP approach may also represent the most convenient option to patients, given the reduced need for polysomnography. The direct-to-autoPAP and unattended approaches merit further attention in an era of patient-centered care that seeks to move more types of care from medical centers to where the patient lives.

The unattended approach resulted in the greatest number of total PSGs for 2 reasons. First, split-night studies are not possible during unattended PSG. Second, as recommended by our external expert panel, we estimated that $20 \%$ of the unattended PSGs would result in unusable data, triggering the need for a subsequent in-laboratory PSG.

Several published studies have used an unattended approach, most notably the Heart Health Study, with excellent results in terms of validity and reliability. ${ }^{16,24,25}$ Studies have reported good to excellent agreement between unattended PSG and laboratory-based PSG (sensitivities, 75-100\%; specificities, 87-100\%). ${ }^{26-28}$ Moreover, some authors have argued that in-home PSGs provide enhanced data when compared with in-laboratory studies because patients feel comfortable sleeping in their own homes, whereas in a sleep laboratory they must acclimatize to an unfamiliar setting, resulting in fewer hours of reliable sleep data. $^{12}$

Unattended PSG is increasingly recognized by national professional organizations. Both the Portable Monitoring Task Force of the American Academy of Sleep Medicine and the VA Sleep-Related Breathing Disorders Source Book refer to unattended PSG as an acceptable alternative when patients cannot accommodate a laboratory-based PSG. ${ }^{10,11}$ The American Academy of Sleep Medicine, in their 2007 Portable Monitoring Task Force Report, recommended the use of portable monitoring when performed under the direction of a certified sleep medicine program for patients with a high likelihood of moderate to severe OSA. ${ }^{11}$ Unattended polysomnography has not yet been 


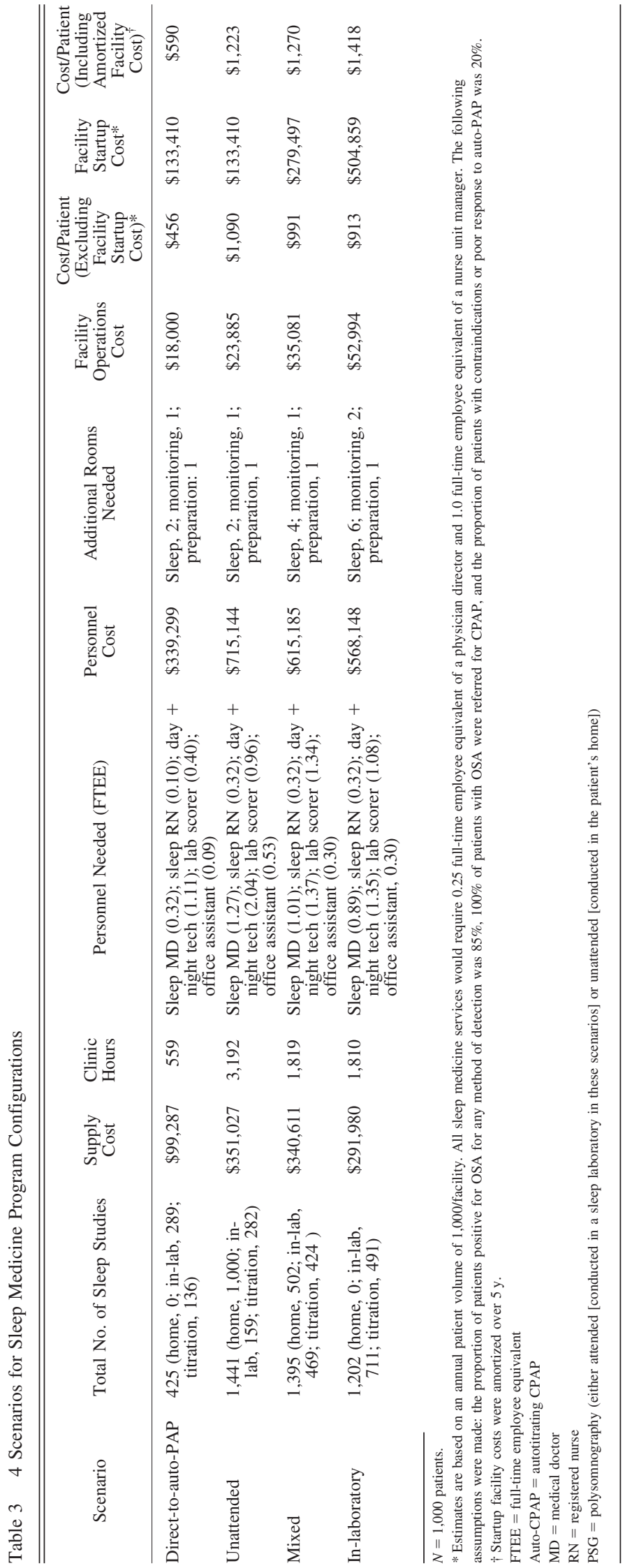


Table 4 Cost Sensitivity to the Rate of Success of Unattended Polysomnography

\begin{tabular}{|c|c|c|c|c|}
\hline \multirow{2}{*}{ Item } & \multicolumn{4}{|c|}{ Success Rate of Unattended Polysomnography } \\
\hline & $50 \%$ & $80 \% *$ & $95 \%$ & $100 \%$ \\
\hline Facility operations & $\$ 31,000$ & $\$ 24,000$ & $\$ 24,000$ & $\$ 24,000$ \\
\hline Personnel & $\$ 826,000$ & $\$ 715,000$ & $\$ 654,000$ & $\$ 633,000$ \\
\hline Supplies & $\$ 404,000$ & $\$ 351,000$ & $\$ 325,000$ & $\$ 316,000$ \\
\hline Clinic hours & 3,360 & 3,192 & 3,108 & 3,080 \\
\hline Total No. of sleep studies & 1,690 & 1,437 & 1,313 & 1,270 \\
\hline Home/Laboratory/Titrations & $1,000 / 388 / 301$ & $1,000 / 155 / 282$ & $1,000 / 39 / 273$ & $1,000 / 0 / 270$ \\
\hline Cost/patient & $\$ 1,261$ & $\$ 1,090$ & $\$ 1,003$ & $\$ 973$ \\
\hline
\end{tabular}

universally adopted in routine clinical practice and may represent a viable approach for many health-care organizations seeking to address unmet demand for diagnosing and treating OSA in a cost-sensitive way. Within the VA, facilities with limited sleep laboratory resources most commonly outsource PSGs to non-VA facilities via a "feebasis" mechanism, with these fee-basis studies almost exclusively consisting of in-laboratory PSGs.

It may be that a single approach to the diagnosis of OSA does not adequately meet the needs of a population served by a given sleep medicine program, especially if the population is diverse in terms of access to a sleep laboratory or preferences about in-home versus in-laboratory testing. The mixed approach provides one relevant scenario of a configuration that allows for local tailoring of sleep medicine services. The mixed approach had per-patient costs that fell between the unattended and in-laboratory approaches. The VA provides an ideal setting in which to develop a tailored sleep medicine approach for several reasons. First, OSA is common among veterans. Second, the VA electronic medical record facilitates both the identification of patients at increased risk of having OSA and communication with primary care providers about sleep medicine recommendations. Third, the VA has relatively few financial barriers for patients to receive care. Fourth, unlike the private sector, which receives less reimbursement from insurance and Medicaid/Medicare for portable monitoring than for in-laboratory polysomnography, practice of care within the VA is less sensitive to thirdparty reimbursement policies.

Attended polysomnography performed in a sleep laboratory remains the accepted standard for diagnosing OSA. There are major barriers, however, associated with scaling up its use to meet current demand. Within the VA, polysomnography may have a long wait time even when using non-VA sleep laboratories under the fee-basis mechanism. Model results indicated that time to treatment initiation was similar for the direct-to-auto-PAP and the unattended approach, twice as long for the mixed approach, and 3 times as long for the in-laboratory approach. Veterans in rural areas often must travel significant distances to the nearest sleep laboratory (VA or non-VA). For some patients, especially those who are caregivers to others, the requirement to stay away from home prevents them from completing an in-laboratory study.

The strength of this study was that the flow model evaluated the relative costs of 4 different configurations of sleep services. Although supply cost estimates were obtained from private-sector United States sources, limitations of this analysis included that personnel and renovation data were all obtained from VA sources and that neither the cost of CPAP machines nor the postage costs for patients to return unattended PSG equipment back to the facility were included in the model. Estimated perpatient costs may thus not generalize to non-VA settings, although the relative differences in costs across scenarios and the key drivers of those costs should still apply. Similarly, because we used salary estimates from Indiana, the estimated per-patient costs are expected to be an overestimate for VA sites with lower locality pay (eg, the South) and an underestimate for VA sites with higher locality pay (eg, New England); however, relative cost differences are again expected to be generalizable. The expenses for unattended polysomnography could be lower than the costs used in this study if equipment is obtained at discounted rates from manufacturers (ie, bulk orders from large health-care systems) and if other cost-saving strategies are used (eg, group visits to instruct many patients in the use of the equipment instead of individual patient instruction). We did not model potential differences in adherence to PAP therapy that may be due to differences in the diagnosis method (eg, patients diagnosed via a direct-to-auto-PAP approach may be less likely to be convinced of their diagnosis of OSA and thereby less inclined to be diligent with PAP therapy) because data regarding the existence or strength of such disparities is currently lacking. Finally, the assumptions used to construct the flow diagrams were based on expert opinion. Therefore, we have been explicit about the assumptions that were included in the study, and although we conducted sensitivity analyses 


\section{Economic Assessment of OSA Diagnosis and Treatment}

related to the performance of the unattended PSGs, other sensitivity analyses could be conducted (eg, changes in the prevalence of OSA).

\section{Conclusions}

Demand for sleep studies far outstrips capacity in traditional facility-based sleep laboratories. Different approaches to diagnosing and treating OSA carry different costs, and this model of 4 different approaches indicated that approaches emphasizing early application of auto-PAP resulted in lower per-patient costs. These findings can assist health-care organizations in assessing tradeoffs and costs when developing their own approaches to addressing unmet needs for diagnosing and treating OSA among their own patient populations.

\section{REFERENCES}

1. Takasaki Y, Orr D, Popkin J, Rutherford R, Liu P, Bradley T. Effect of nasal continuous positive airway pressure on sleep apnea in congestive heart failure. Am Rev Respir Dis 1989;140(6):1578-1584.

2. Finkel KJ, Searleman AC, Tymkew H, Tanaka CY, Saager L, SaferZadeh E, et al. Prevalence of undiagnosed obstructive sleep apnea among adult surgical patients in an academic medical center. Sleep Med 2009;10(7):753-758.

3. Singh M, Liao P, Kobah S, Wijeysundera DN, Shapiro C, Chung F. Proportion of surgical patients with undiagnosed obstructive sleep apnoea. Br J Anaesth 2013;110(4):629-636.

4. Wolfe RM, Pomerantz J, Miller DE, Weiss-Coleman R, Solomonides T. Obstructive sleep apnea: preoperative screening and postoperative care. J Am Board Fam Med 2016;29(2):263-275.

5. Young T, Peppard P, Gottlieb DJ. Epidemiology of obstructive sleep apnea: a population health perspective. Am J Respir Crit Care Med 2002;165(9):1217-1239.

6. Yaggi HK, Concato J, Kernan WN, Lichtman JH, Brass LM, Mohsenin V. Obstructive sleep apnea as a risk factor for stroke and death. N Engl J Med 2005;353(19):2034-2041.

7. Colten H, Abboud F, Block G, Boat T, Litt I, Mignot E, et al. Sleep Disorders and sleep deprivation: an unmet public health problem. Washington, DC: National Academy of Sciences; 2006:217-232.

8. Flemons WW, Douglas NJ, Kuna ST, Rodenstein DO, Wheatley J. Access to diagnosis and treatment of patients with suspected sleep apnea. Am J Respir Crit Care Med 2004;169(6):668-672.

9. Flemons WW, Douglas NJ, Kuna ST, Rodenstein DO, Wheatley J. Access to diagnosis and treatment of patients with suspected sleep apnea. Am J Respir Crit Care Med 2004;169(6):668-672.

10. Hirshkowitz M, Littner M, Kuna S, Berry R, Norris M, Almenoff P. Sleep-related breathing disorders: sourcebook. 2nd Edition. Milwaukee, Wisconsin: Healthcare Analysis and Information Group (HAIG), VHA; 2003.

11. Collop NA, Anderson WM, Boehlecke B, Claman D, Goldberg R, Gottlieb DJ, et al. Clinical guidelines for the use of unattended portable monitors in the diagnosis of obstructive sleep apnea in adult patients. J Clin Sleep Med 2007;3(7):737-747.

12. Iber C, Redline S, Kaplan Gilpin A, Quan SF, Zhang L, Gottlieb DJ, et al. Polysomnography performed in the unattended home versus the attended laboratory setting: Sleep Heart Health Study methodology. Sleep 2004;27(3):536-540.

13. Massie CA, McArdle N, Hart RW, Schmidt-Nowara WW, Lankford A, Hudgel DW, et al. Comparison between automatic and fixed positive airway pressure therapy in the home. Am J Respir Crit Care Med 2003;167(1):20-23.

14. Fitzpatrick MF, Alloway CE, Wakeford TM, MacLean AW, Munt PW, Day AG. Can patients with obstructive sleep apnea titrate their own continuous positive airway pressure? Am J Respir Crit Care Med 2003;167(5):716-722.

15. Kuna S. Portable-monitor testing: an alternative strategy for managing patients with obstructive sleep apnea. Respir Care 2010;55(9): 1196-1215.

16. Bravata DM, Ferguson J, Miech EJ, Agarwal R, McClain V, Austin $\mathrm{C}$, et al. Diagnosis and treatment of sleep apnea in patients' homes: the rationale and methods of the "GoToSleep" randomized-controlled trial. J Clin Sleep Med 2012;8(1):27-35.

17. Netzer NC, Stoohs RA, Netzer CM, Clark K, Strohl KP. Using the Berlin Questionnaire to identify patients at risk for the sleep apnea syndrome. Ann Intern Med 1999;131(7):485-491.

18. Srijithesh PR, Shukla G, Srivastav A, Goyal V, Singh S, Behari M. Validity of the Berlin Questionnaire in identifying obstructive sleep apnea syndrome when administered to the informants of stroke patients. J Clin Neurosci 2011;18(3):340-343.

19. Bravata DM, Concato J, Fried T, Ranjbar N, Sadarangani T, McClain V, et al. Auto-titrating continuous positive airway pressure for patients with acute transient ischemic attack: a randomized feasibility trial. Stroke 2010;41(7):1464-1470.

20. Bravata DM, Concato J, Fried T, Ranjbar N, Sadarangani T, McClain V, et al. Continuous positive airway pressure: evaluation of a novel therapy for patients with acute ischemic stroke. Sleep 2011; 34(9):1271-1277.

21. Pietzsch JB, Garner A, Cipriano LE, Linehan JH. An integrated health-economic analysis of diagnostic and therapeutic strategies in the treatment of moderate-to-severe obstructive sleep apnea. Sleep 2011;34(6):695-709.

22. Chervin RD, Murman DL, Malow BA, Totten V. Cost-utility of three approaches to the diagnosis of sleep apnea: polysomnography, home testing, and empirical therapy. Ann Intern Med 1999;130(6):496-505.

23. Kim RD, Kapur VK, Redline-Bruch J, Rueschman M, Auckley DH, Benca RM, et al. An economic evaluation of home versus laboratorybased diagnosis of obstructive sleep apnea. Sleep 2015;38(7):1027-1037.

24. Redline S, Sanders MH, Lind BK, Quan SF, Iber C, Gottlieb DJ, et al. Methods for obtaining and analyzing unattended polysomnography data for a multicenter study. Sleep Heart Health Research Group. Sleep 1998;21(7):759-767.

25. Kapur VK, Rapoport DM, Sanders MH, Enright P, Hill J, Iber C, Romaniuk J. Rates of sensor loss in unattended home polysomnography: the influence of age, gender, obesity, and sleep-disordered breathing. Sleep 2000;23(5):682-688.

26. de Oliveira ACT, Martinez D, Vasconcelos LFT, Cadaval Gonçalves S, do Carmo Lenz M, Costa Fuchs S, et al. Diagnosis of obstructive sleep apnea syndrome and its outcomes with home portable monitoring. Chest 2009;135(2):330-336.

27. Zou D, Grote L, Peker Y, Lindblad U, Hedner J. Validation a portable monitoring device for sleep apnea diagnosis in a population based cohort using synchronized home polysomnography. Sleep 2006; 29(3):367-374.

28. Li CK, Flemons WW. State of home sleep studies. Clin Chest Med 2003;24(2):283-295. 